\title{
Research on copyright appointment registration microplatform system based on vue
}

\author{
Mengdie $\mathrm{Gu}^{1, *}$, Rui $\mathrm{Sun}^{2}$, Shulin $\mathrm{Yang}^{1}$, Huijie $\mathrm{Gu}^{1}$ and Ming Yuan ${ }^{3}$ \\ ${ }^{1}$ Electronic and Communication Engineering, Beijing Institute of Graphic Communication \\ ${ }^{2}$ Information Support Room, Air Force Armament Department \\ ${ }^{3}$ Information and Communication Engineering, Beijing Institute of Graphic Communication
}

\begin{abstract}
With the rapid development of copyright industry, people's copyright awareness of their own creation of software and works is constantly improving, the number of applications for software copyright registration and works copyright is increasing. Therefore, how to make copyright registration business becomes more convenient and efficient is imminent. Existing copyright registration system need to first fill through $\mathrm{PC}$ and then make an appointment through the phone to deal with relevant business. This model is not only complicated operations, but also poor user experience. Therefore, based on the analysis of multiple needs of users and enterprises, this paper proposes to use Vue,SpringBoot, MyBatis and other technologies to develop a copyright appointment registration system based on microplatform. The development of this system can not only for the user to create a more convenient way of operation, give users a better experience, but also can change, rich business approach, bring more profits.
\end{abstract}

Keywords: Vue, copyright appointment registration, SpringBoot, microplatform.

\section{Introduction}

The continuous development of information technology has lowered the threshold for people to access Internet resources. In this era of whole-network information sharing, the public can easily see others' works and save them online ${ }^{[1]}$. Therefore, people's awareness of the copyright of their own software and works is constantly improving, and it is very common to prove the ownership of their works by applying for software copyright registration and works copyright registration. As the only national copyright registration organization, the copyright registration system undertakes all software copyright registration tasks and part of the work copyright registration tasks. The system has played a great role in promoting the development of copyright registration business. According to the analysis report on software copyright registration in China in 2020 issued by China Copyright Protection Center on March 18, 2021, it is pointed out that in 2020, China registered a total of 1722,904 computer software Copyrights, an increase of nearly 240,000

\footnotetext{
*Corresponding author: 2643963632@qq.com
} 
compared with that in 2019 , basically continuing the registration trend of substantial growth since 2017, with the total number of registrations increasing by more than 200,000 annually for four consecutive years ${ }^{[2]}$.You can see that the copyright registration business shows rapid growth trend. With the continuous improvement of the copyright registration business, the number of people handling the business continues to increase, users' requirements for the quality of copyright registration service are also increasingly high. Both the convenience of registration and appointment and the operability and aesthetics of the system have become an important indicator for people to measure the service level of the copyright industry. However, the current mode of copyright registration and filing through PC terminal and booking through mobile terminal in the Copyright Center can no longer meet the users' demand for fast and efficient handling of copyright services. In this environment, a new way to solve the current problems needs to be explored.

In addition, with the rapid development of $4 \mathrm{G} / 5 \mathrm{G}$ technology, the operation mode of mobile terminals is becoming more and more popular with the public. The way of online operation on PC can no longer meet people's requirements for the convenience of study and life. The emergence of mobile APP makes it easy for people to work online without carrying heavy terminals. However, this way not only requires downloading the corresponding software to carry out the corresponding operation, but also brings some security risks. The emergence of the "micro" era has broken these shackles. WeChat official account is used to handle business without carrying bulky computer or downloading any software. You can enter the copyright appointment registration system and conduct corresponding operation according to the identity authority only by paying attention to it. In this way, users can understand the information of copyright registration regardless of time and region, complete the operation of registration and appointment, eliminate the tedious process of filling on the $\mathrm{PC}$ and booking on the mobile end, bring users a more convenient way of registration and filling in, and improve users' satisfaction with the copyright industry.

Therefore, in order to solve the above problems, this paper proposes a copyright appointment registration system based on WeChat public account. The design and implementation of this system is an innovation of the existing service management mode of the copyright industry, and a breakthrough of the single terminal for its business management. The research and implementation of appointment registration on microplatform will provide theoretical support for promoting the transformation and development of the copyright industry. The development of microplatform not only enables users to conduct business operations more convenient and efficient, improve user satisfaction, but also enhances the business volume of enterprises and brings greater profits to enterprises.

\section{The implementation principle and advantages of Vue.js technology}

As Web applications become more and more complex, front-end frameworks such as Angular, React, and Vue have become the first choice for most developers to develop frontend pages. This project will choose Vue.js technology, which is very popular in current development, for front-end development. Compared with the Angular and React front-end frameworks, Vue.js is a progressive framework for building user interfaces and a JavaScript library based on the MVVM pattern ${ }^{[3]}$. It has bi-directional data binding, modularization, componentization, plug-in, lightweight framework and other features, can help developers quickly build pages and reduce the amount of code.It can also be quickly integrated with third-party plug-ins to simplify complex applications and improve development efficiency. Vue framework has been widely used in many fields of systems, such as the 
implementation of university online education system, bank front desk system, etc ${ }^{[4]}$.

The development of two-way data binding is the core of Vue.js technology. Bidirectional data binding enables automatic data interaction between data sources and DOM [5]. This is shown in Figure 1. Meanwhile, Vue.js realizes two-way data binding in combination with the observer subscriber pattern, and its schematic diagram is shown in Figure 2. The realization of data two-way binding is mainly divided into two processes. When the data in the new Vue () instance object is passed a property, the property is written to a DEP empty object, each property is one-to-one corresponding to its DEP, and the get and set methods of all properties are listened on. The instructions in the view, on the other hand, are parsed, and each instruction creates a Watcher and passes the Watcher to the DEP object for the corresponding property. When Observer monitors the change of an attribute, notify method in the data Dep object will be called to traversal the corresponding Watcher, and the Update method of Watcher will be called to update the view, so as to realize the responsive update of data. At the same time, if Vue listens for DOM changes, it will also modify the corresponding data. The responsive data binding forms the data-view-change mode, which greatly improves the development efficiency of the system.

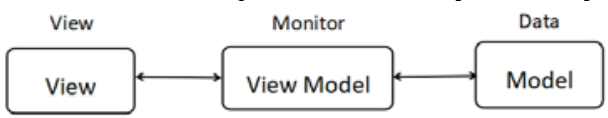

Fig. 1. Progressive frame structure diagram.

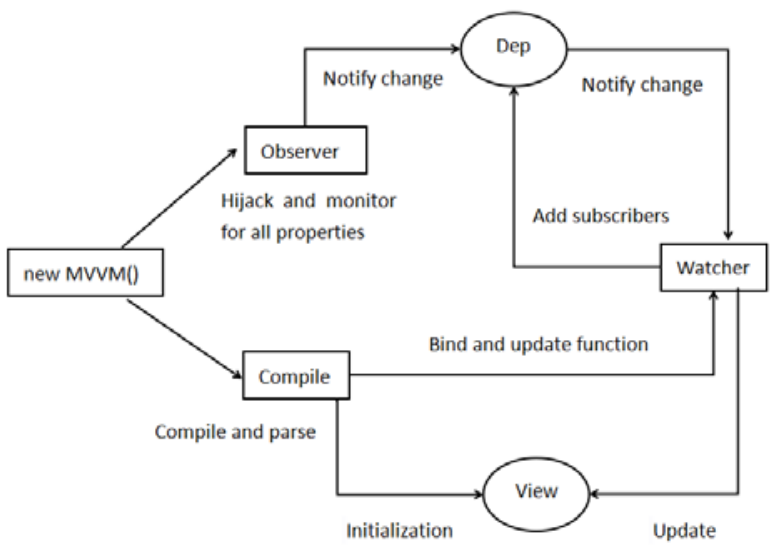

Fig. 2. Schematic diagram of two-way data binding.

Componentization is also one of the strengths of the Vue framework. In a Vue project, pages are usually divided separately according to business or module, a complete page is divided into independent components, and components in a page are integrated in a folder, so as to facilitate the management of the same page ${ }^{[6]}$. At the same time, each component contains the view template temple in the component, the initial data of the component, the parameters received props, the methods used in the component, life cycle functions, CSS, etc, all the information in the component is gathered in a .Vue file, which is easy to maintain in the later stage. If a component wants to be used on multiple pages or projects at the same time, it can be reused on multiple pages simply by encapsulating the component as a single component and using it in conjunction with slot slots. In use, only need to replace the slot in the page with the required content to achieve the corresponding page, reducing the developers to write repeated code, improving the efficiency of development.

Vue also has richer third-party plug-ins than Angular or React. In addition to using componentized development idea, this project also uses Vue's Vue-Router, Vuex, Axios and other third-party plug-ins. Vue-Router is the official routing plug-in for Vue.js, which 
can be deeply integrated with Vue.js for building single-page applications.Vue single-page application is based on routing and components. Routes are used to set access paths and map paths and components to achieve page jump, without the need of switching and jumping pages through hyperlinks and defining multiple .html files like traditional pages ${ }^{[7]}$. Vuex is used to manage the state of the program. Vuex uses centralized storage to manage the state of all components. For views that depend on the same state, supporting multiple views to change the same state can greatly reduce the coding of repeated code and improve development efficiency. The core Store of the Vuex application contains five core functions: State, Getter, Mutation, Action, and separating the Store into modules, which can change the data State synchronously or asynchronously. The project also uses Axios to send network requests to the server. Axios is a Promise-based HTTP library that creates XMLHttp Requests from the browser, supports the Promise API, intercepts requests and responses, transforms request data and response data, cancels requests, and automatically transforms JSON data to facilitate system development ${ }^{[8]}$.To sum up, based on these characteristics of Vue.js technology, it is the best choice to use Vue to develop the front page of the copyright appointment registration system.

\section{Design of copyright appointment registration microplatform system}

\subsection{The general design idea of copyright appointment registration microplatform system}

The copyright appointment registration microplatform system is mainly divided into two parts: the client side mainly contains two roles: individual user and enterprise user, and the management side mainly carries out related operations for the administrator. The functions that individual users and enterprise users can use when entering the system are divided into modules,including registration and login module, registration application module, appointment module, registration announcement module and individual center module. The design and implementation of these modules can enable users to complete the registration, filling and appointment of software and works copyright through a single mobile phone terminal regardless of time and region. At the same time, the latest information of registration and appointment issued by the system can be timely understood, and the information of copyright registration applied for and the progress of business can be easily and quickly inquired. The administrator can maintain and manage the data of the whole system. The main functions include reviewing the submitted copyright registration application to ensure the authenticity, legality and uniqueness of the works, User management module, appointment management module, content management module and system management module.This system uses HTML5, CSS, Vue.js and Vant-UI technology to complete the development of the front end, Spring Boot, MyBatis technology to complete the development of the back end and MySQL database.The above technologies are integrated to achieve the development of copyright appointment registration microplatform system.

\subsection{Function design of copyright appointment registration microplatform system}

Copyright appointment registration microplatform system is the user and the copyright industry an important bridge.Its research and design can not only solve the user's trouble through the multi-terminal appointment registration business, simplify the registration 
appointment process, improve the efficiency of the copyright registration, improve user satisfaction to copyright service industry, also can bring more customers resources and profits to the enterprise, lay a solid foundation for the future development of the enterprise. The functional structure of the microplatform system for copyright appointment and registration is shown in Figure 3.

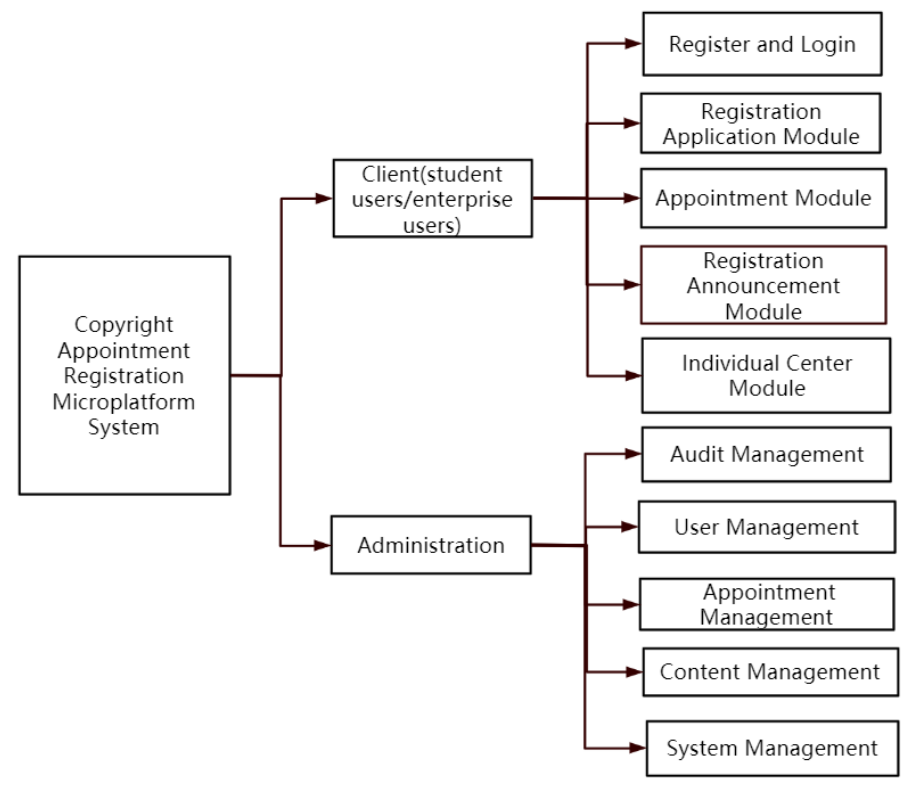

Fig. 3. Function structure diagram of copyright appointment registration microplatform system.

\subsubsection{Client}

User registration and login module: both individuals and enterprises need to register and log in before entering the corresponding permissions page and carrying out relevant operations. If it is an individual user, you need to fill in the information including name, ID number, mobile phone number, mobile phone verification code, email, account number, password and other information to register. After verification at the background, you can enter the page of personal user permissions. If the user is an organization, the user needs to upload the company name, company address, company legal person name, legal person ID number, legal person mobile phone number, mobile phone verification code, email, business license photo and other enterprise certificates. The background administrator verifies the authenticity and legality of the enterprise identity, evaluates the credit value of the enterprise, and then issues the authorization. After the registration is completed, the user can choose different roles to log in. If the user forgets the password, the user can retrieve the password through mobile phone number or email.

Registration application module: Users can apply for copyright registration after logging in. This module is divided into two parts: application for software copyright registration and application for copyright registration of works. The application for software copyright registration includes six businesses, such as application for registration of computer software copyright transfer or proprietary license contract,change or supplement of software registration items, and request for cancellation of computer software registration. The application for copyright registration of works includes 18 services, such as application for the record of copyright contract, copyright-related rights registration application, contract registration application for reprinting foreign periodicals, 
copyright of works and contract modification or supplementary registration application. Users can complete the registration application by filling in relevant information in the business they need to choose.

Appointment module: For users who have completed the application for copyright registration, they can choose the appropriate date and time period to make an appointment for copyright-related business in this module, and they can also check the specific appointment process and appointment instructions.

Registration announcement module: for the approved copyright registration application, you can query the detailed information of the software or work by inputting the registration number, name of the software or work, registration time and other information in this module.

Individual center module: this module includes user information, my appointment, progress query, stream number checking, account switching, logout and other functions. It will also show how many registrations the user has made and how many applications are pending.

\subsubsection{Administration}

Audit management module: the administrator shall review the authenticity, legality, uniqueness and other contents of the submitted application data for copyright registration, and give the judgment of approval and rejection. If the examination is passed, a certificate shall be issued to the copyright owner to determine his ownership of the work. If the audit is not successful, the result will be shown in the progress of the registration process in the user center.

User management module: user management mainly includes individual user management and enterprise user management. Administrators can add, delete, change, check and allocate permissions to users.

Content management module: this module will carry on the centralized and unified management to the text and picture content of all pages, including the operation of adding, deleting, modifying and checking the picture content and text content. For example: the microplatform page of the rotation of pictures, information announcement content, etc.

Appointment management module: the administrator can set the number of appointments on the platform every day and whether the appointment can be made on the day according to the time period. The design of this module helps to disperse the number of registrations and ensure the orderly operation of the business.

System Management module: The copyright appointment and registration system not only contains important personal information but also contains the core content of the software and works created by users. Therefore, the login personnel of the system should be more closely monitored to ensure the security of data and information. This module mainly supervises the personnel who log into the system. When the personnel log into the system, the login log will record the user ID, user name, time and other contents of the login, so as to realize the purpose of restricting the behavior of the system operator and ensuring the safety of the system operation.

\subsection{The general architecture and analysis on key technologies of the copyright appointment registration microplatform system}

The front end of the system will mainly use Vue, HTML5, CSS, Vant-UI and other technologies. The back end will mainly use Spring Boot, MyBatis technology for development. Users can pay attention to the WeChat public account on their 
mobile phones to access the the copyright appointment registration microplatform system. The system architecture is shown in Figure 4.

$$
\text { Access Layer } \Longrightarrow \begin{aligned}
& \text { Enter the copyright appointment registration microplatform system } \\
& \text { through WeChat public account }
\end{aligned}
$$
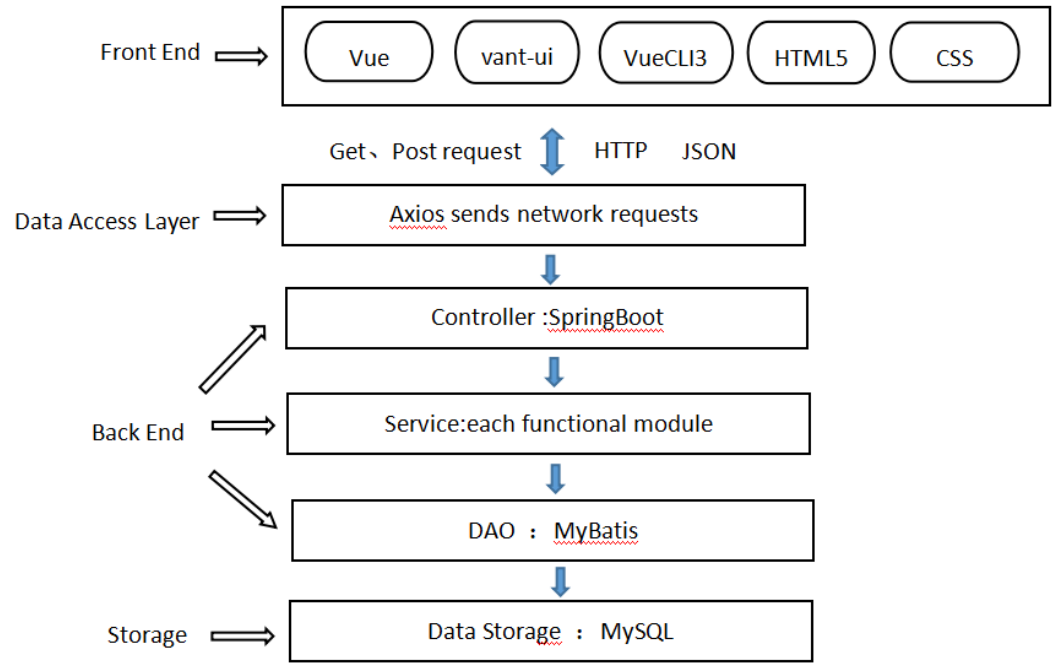

Fig. 4. System architecture diagram of copyright appointment registration microplatform.

\subsubsection{The front end}

The front-end of this project will be developed using the currently popular Vue.js framework. By installing Vuecli3 scaffolding to help build and manage the project, Vuecli3 can automatically generate the project directory structure and Webpack and other relevant configurations, simplifies the configuration of the project and improves the development efficiency. At the same time, the front-end will use the vant-UI component library's rotation diagram, pop-up box, icon, button and other components to build the page of the system. Vant-UI is a multi-functional component library developed based on VUE, which mainly serves the mobile terminal business. It has rich UI components, which can effectively simplify the amount of code development and make the interface layout more reasonable and beautiful. In addition, in order to solve the problem that too many images appear blank on the interface, this project also uses the image lazy loading component V-lazy in the Vant-UI component library. The use of V-lazy will reasonably allocate the loading order of images in the visible area and the non-visible area, that is, pictures appearing in the visible area will be loaded in priority. This can greatly enhance the user's visual effect and experience.

\subsubsection{Data access layer}

The data access layer will make network requests using Axios, which is officially recommended by Vue, and complete the network requests by configuring the server's URL, params and other parameters. When the request is successful, the then method is called to get the requested data and process the data accordingly. When the page sends a network request to the server, the server will return the data of the whole page at one time, and then 
distribute the content of the component traversing through the V-for instruction to each subcomponent for display, instead of making a network request for each operation of the subcomponent, which greatly reduces the server's access and avoid data delays caused by too many server accesses. When concurrent requests occur on a page, Axios's all method is called to process multiple requests simultaneously, and the result is returned as an array when multiple network requests have been completed.

\subsubsection{Back end and storage layer}

The backend of this project is designed and implemented using the currently popular SpringBoot+ MyBatis framework, and adopts the hierarchical architecture mode of control layer, business logic layer and data access layer. The adoption of this architecture mode can make the code structure more clear, reduce the dependency between layers, and improve performance and scalability [9]. In the later maintenance time, it can also greatly reduce the maintenance cost and maintenance time. MySQL database is used for data storage. MySQL is a middleware that integrates SQL addition, deletion, check and change as well as a large number of advanced database operations. It encapsulates all operations of JDBC, and can quickly generate codes through simple XML configuration and annotations, which can greatly improve the productivity of developers. Moreover, MySQL database has good security and connectivity [10].

\section{Conclusion}

On the basis of analyzing the problems existing in the existing copyright appointment registration system, this paper proposes to develop the copyright appointment registration microplatform system based on Vue.js technology with WeChat public account as the carrier. This system enables users to register and make appointment in copyright more convenient and quick without being restricted by terminals and regions. It can not only enhancing users' experience, users' overall satisfaction with the copyright industry, but also increase the business volume of the Copyright Center and bring more users and profits to the Copyright Center. It can be said that the development of this project will build a new communication bridge between copyright business management and users, and help corporate users and individual users to quickly and efficiently complete the requirements of copyright registration and appointment. However, the extensibility and safety of this system need to be improved in the later research process.

The authors would like to thank the Fund for the Research on Key Technologies of Intelligent Fault Management Platform for Printing Equipment (Eb202103) for their support in this research.

\section{References}

1. Jingyi Tian. Research on China's Digital Picture Copyright Protection under the Background of Whole Network Sharing [D]. Sichuan International Studies University,2019.

2. China Copyright Protection Center. Analysis Report on Software Copyright Registration in 2020 in China. https://www.ruanfujia.com/239732/, 2021-03-22.

3. Zhiren Wang. Design and Implementation of Development Platform Based on Vue.js [D]. Guangdong University of Technology,2018. 
4. Junhui Song,Min Zhang,Hua Xue. Design and Implementation of a Vue.js-Based College Teaching System[J].International Journal of Emerging Technologies in Learning (iJET),2019,14(13):59-69.

5. Bin Hu. New Energy Vehicle Remote Upgrade Management Platform Based on VUE Framework [J]. Information Technology and Informatization,2020(08):45-47.

6. Wenwen Pan. Research and Implementation of Several Optimization Technologies for Web Front-end on Cloud Platform [D]. University of Electronic Science and Technology of China,2020.

7. Ning Sun,Weiping Zhao,Yufei Wang,et al.Design and Implementation of Chinese Ancient Spectral Database Management Platform [J]. Modern Computer (Professional Edition),2017(1):64-69.

8. Erhua Zhu.Research on Web Front-end Application Based on Vue.js [J]. Science Technology and Innovation,2017, 20:119-121.

9. Jiaqi Zhu. Design and Implementation of Video Surveillance Networking and Sharing Platform [D]. Beijing Jiaotong University,2019.

10. JEROEN O,DAVID J,SAIKAT D,et al.RMySQL:database interface and 'MySQL' driver for $\mathrm{R}[\mathrm{J}] .2018$. 